\title{
High speed imaging of solid needle and liquid micro-jet injections
}

Cite as: J. Appl. Phys. 125, 144504 (2019); https://doi.org/10.1063/1.5074176

Submitted: 22 October 2018 . Accepted: 20 March 2019 . Published Online: 09 April 2019

Loreto Oyarte Gálvez (D), Maria Brió Pérez (D), and David Fernández Rivas (D)

\section{COLLECTIONS}

F This paper was selected as Featured
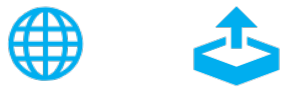

\section{ARTICLES YOU MAY BE INTERESTED IN}

Terahertz waveform considerations for nonlinearly driving lattice vibrations

Journal of Applied Physics 125, 144101 (2019); https://doi.org/10.1063/1.5052638

Features of streamer formation in a sharply non-uniform electric field

Journal of Applied Physics 125, 143301 (2019); https://doi.org/10.1063/1.5067294

A multifunctional molecular spintronic platform with magnetoresistive and memristive responses via a self-assembled monolayer

Journal of Applied Physics 125, 142905 (2019); https://doi.org/10.1063/1.5057893

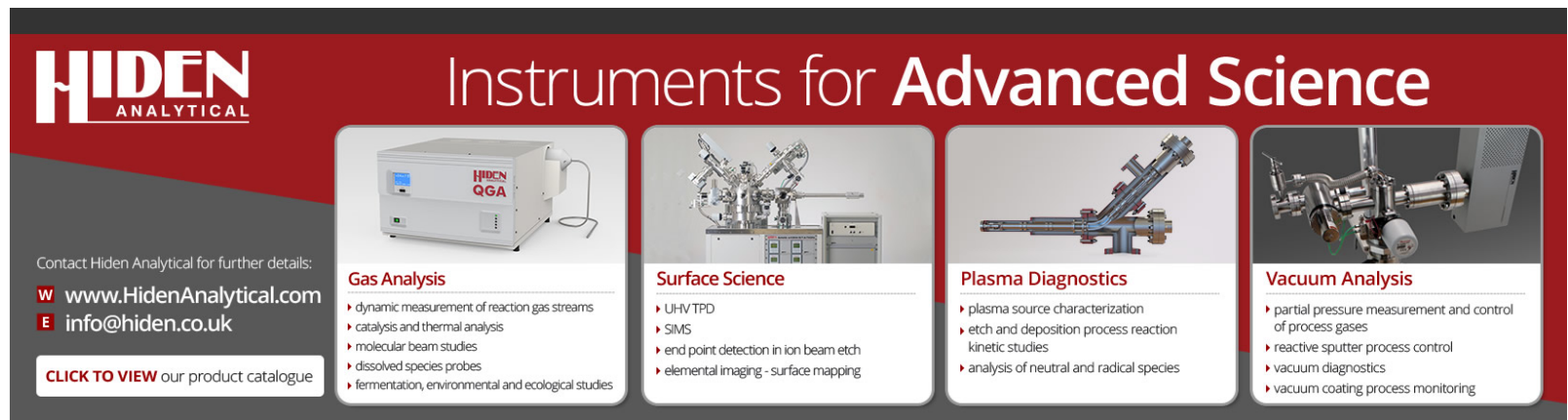




\title{
High speed imaging of solid needle and liquid micro-jet injections
}

\author{
Cite as: J. Appl. Phys. 125, 144504 (2019); doi: 10.1063/1.5074176 \\ Submitted: 22 October 2018 . Accepted: 20 March 2019. \\ Published Online: 9 April 2019

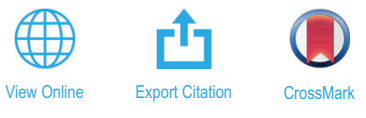

Loreto Oyarte Gálvez, a),b) (iD Maria Brió Pérez, (D) and David Fernández Rivas (D)

AFFILIATIONS

Mesoscale Chemical Systems Group, MESA+ Institute and Faculty of Science and Technology, University of Twente, Enschede 7522NB, The Netherlands

a) Present address: Department of Ecological Science, Vrije Universiteit Amsterdam, Amsterdam 1081HV, The Netherlands.

b).oyartegalvez@gmail.com

\begin{abstract}
We have used high speed imaging to capture the fast dynamics of two injection methods. The first one and perhaps the oldest known is based on solid needles and used for dermal pigmentation, popularly known as tattooing. The second is a novel needle-free microjet injector based on thermocavitation. Injections in agarose gel skin surrogates were made with both methods and ink formulations having different fluidic properties. Water, a glycerin-water mixture, and commercial inks were used with both injectors to understand better end-point injection. The agarose deformation process due to the solid needle injection helped establish an assessment of penetration potential by using the dimensionless penetration strength quantity. We found that microjet injections are superior than solid injections in terms of energy and volumetric delivery efficiencies per injection for three different liquids. The microjet injector could reduce the environmental impact of used needles and benefit millions of people using needles for medical and cosmetic use.
\end{abstract}

Published under license by AIP Publishing. https://doi.org/10.1063/1.5074176

\section{INTRODUCTION}

Tattooing, also known as dermal pigmentation, is done by inserting exogenous substances such as pigments into the dermis and leaving a permanent mark. ${ }^{1-3}$ The earliest evidence of tattooing procedures traces back to the fourth millennium B.C.E. ${ }^{4}$ As evidenced by mummified skin, ancient art, and the archaeological records, tattoos have served two basic functions: medicinal or cosmetic. ${ }^{5}$ Two distinctive types of cosmetic tattoos exist: the conventional or purely decorative, and permanent make-up intended to alleviate existing conditions, e.g., scar camouflaging, alopecia, or postmastectomy pigmentation of a nipple on cancer patients.

The societal acceptance of tattoos and permanent make-up has widened, with a worldwide increase in its numbers and the social groups having them. In Europe, $12 \%$ of the population has one or more tattoos, ${ }^{6}$ corresponding to more than $44 \times 10^{6}$ tattooed Europeans; the figures in the U.S.A. and other countries are similar or higher. According to experts, depending on the skin type and individual, a tattoo can be painful and cause skin related allergies, while $20 \%-50 \%$ of the ink is not injected. ${ }^{7}$ Hence, our society as a whole will be posed with scientific and technological challenges to reduce health risks caused by tattooing and palliate its economic consequences.
The millenarian method of tattooing and permanent make-up is in principle the same: the repeated insertion of one or several needles into the skin delivers ink droplets through the open wounds. Solid needles with single or multiple tips are typically sold as single-use consumables. The ink that adheres to the needle surface is transported into the skin dermis depending on the angle of the needle with respect to the skin and the pressure applied by the tattoo artist or the cosmetic technician. The exact ink formulation is kept as a secret by commercial brands, with ingredients roughly categorized according to function. Inks are composed of different pigments suspended in a carrier solution, together with binders and additives. ${ }^{6}$ Each ink has different ingredient proportions, which enable tailored fluid dynamic properties, such as viscosity, surface tension, and density. ${ }^{7,8}$ Pigments are inorganic particles responsible for the ink visual perceptible color, with a particle size range of $0.1 \mu \mathrm{m}-50 \mu \mathrm{m}$. The larger the particle, the harder for the immunological system of the human body to remove it, resulting in more permanent tattoos. ${ }^{9}$

Tattoo machines have been adapted for intradermal injections to evenly inject into a large area of the skin, dividing effectively the dose in smaller portions. ${ }^{10}$ In recent decades, alternative cutaneous 
delivery methods have been developed, including high pressure injections, ${ }^{11}$ needle-assisted jet injectors, ${ }^{12}$ as well as microneedles and biodegradable structures. ${ }^{13,14}$ A liquid jet injector is a needlefree medical device that pressurizes thin liquid filaments to penetrate the skin, with clear advantages over conventional injections with needles. ${ }^{10,15-17}$ Jet injectors were used in smallpox eradication, preventing rabies, influenza, malaria, hepatitis $\mathrm{A}$ and $\mathrm{B}$, injecting insulin, and analgesics. ${ }^{10,16-20}$ Some proven advantages of jet injectors over conventional needles are higher immunological response, drug dose sparing, reduction in pain with improved patient compliance, and reduction of accidental needle-stick incidents. ${ }^{21}$

Lasers can be used to heat a liquid above its boiling point with an explosive phase transition, with varied applications besides injecting, e.g., small droplets dispensing and patterning. ${ }^{22-25}$ If a continuous wave $(\mathrm{CW})$ laser is used as an energy source, this phenomenon is known as thermocavitation. ${ }^{26,27}$ The most recent report on needle-free injection using thermocavitation relies on a fast growing vapor bubble inside a microfluidic channel that provides confinement. ${ }^{28,29}$ To the best of our knowledge, a rigorous description of the tattooing process-one of the oldest transdermal injection methods-is presented for the first time. A comparison with a thermocavitation microjet injector device-as a needle-free injection method-is performed on the basis of quantification of different observables, as well as operation parameters of relevance for applications such as potential damage to the skin, power required for injecting, and energy efficiency per injection.

\section{EXPERIMENTAL SETUP AND PROCEDURE}

\section{A. Solid needle injector}

A solid needle injector was vertically fixed to inject inks into skin surrogates made of agarose gel [see Fig. 1(a)]. This injector is a pigmentation instrument (PL-1000 Mobil, PERMANENT-Line $\mathrm{GmbH}$ ), composed of an electric control unit, a hand piece, and a consumable hygiene needle module. The hand piece includes a motor that moves the attached needle up and down in a smooth, cyclical pattern. ${ }^{30}$ The control unit enables an adjustable nominal injection frequency in the range $f_{n}=[50-150] \mathrm{Hz}$. A disposable single-use module is attached to the hand piece, containing a sterilized stainless-steel solid needle with a diameter of $D_{\text {needle }}=4 \mathrm{~mm}$.

The agarose gel samples were placed in a custom-made holder, under the hand piece, with the gel surface almost touching the needle tip, as shown in Fig. 1. The gel is confined by glass transparent walls providing a depth ( $y$-axis), width ( $x$-axis), and length ( $z$-axis) of $\sim 3 \mathrm{~mm}, 3 \mathrm{~mm}$, and $20 \mathrm{~mm}$, respectively. A mirror with a $45^{\circ}$ orientation is placed below the gel. This system allows the simultaneous frontal and bottom visualization of the injection processes into the gels. The holder is attached to a one-axis motorized translation stage (MT1/M-Z8, Thorlabs), which allows one to keep the gel stationary or in motion at a constant speed $v_{\text {motor }}=2 \mathrm{~mm} / \mathrm{s}$, along a plane perpendicular to the needle.

Front view images were made with a color high-speed camera (Fastcam SA2, Photron) capturing 1000 frames per second (fps); see Fig. 1(b). In addition, the agarose gel deformation was measured with dry needles-without ink-using a monochromatic high-speed camera (Fastcam SA-X2, Photron) recording at $10000 \mathrm{fps}$ and with a high-spatial resolution of 1409 pixels per
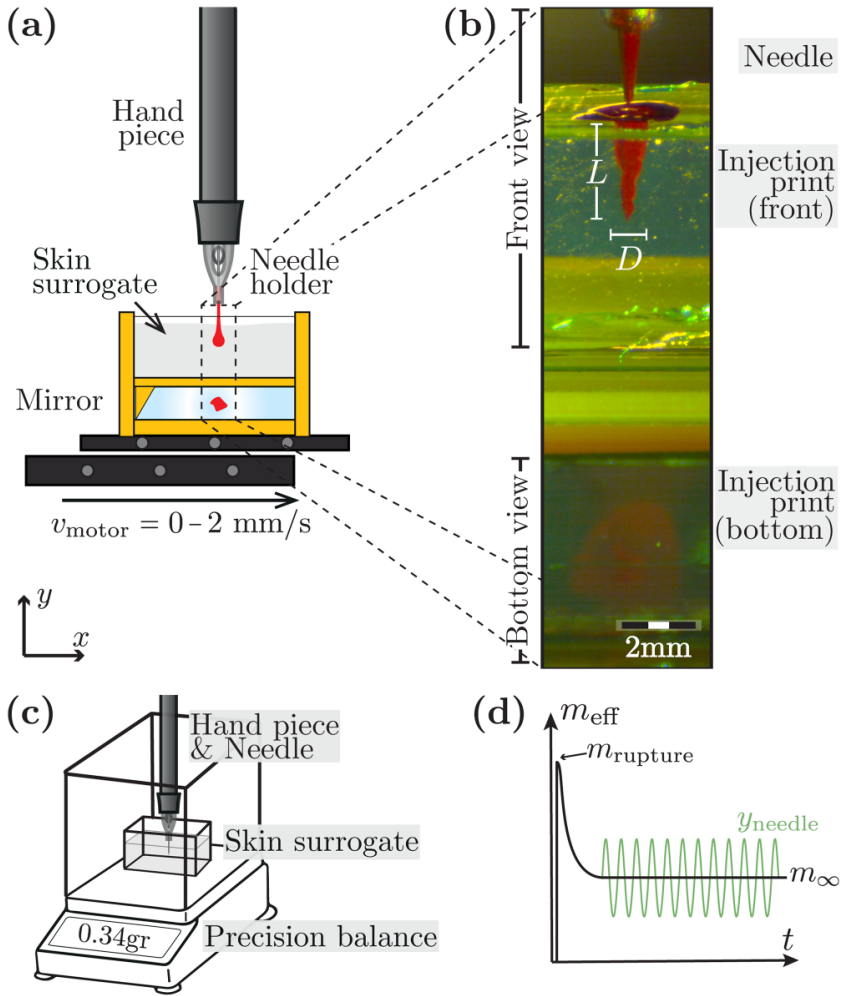

FIG. 1. (a) Schematics of the solid needle injector setup: the hand-piece is vertically fixed onto which a solid needle holder is attached. The agarose gel skin surrogates were located below and almost touching the needle tip at rest. The agarose gel can be kept stationary $\left(v_{\text {motor }}=0\right)$ or in motion $\left(v_{\text {motor }}=2 \mathrm{~mm} / \mathrm{s}\right)$, along a plane perpendicular to the needle. A high-speed camera recorded the injection process at $1000 \mathrm{fps}$. (b) Example of images obtained with the highspeed camera: the injection length $L$ and diameter $D$ are determined from the front view, whereas the bottom view shows the ink spread around the needle. (c) Experimental setup used to measure the force exerted on the skin surrogates with a precision balance. (d) The initially measured peak mass $m_{\text {rupture }}$ corresponds to the rupture of the gel due to the needle penetration. After that, the balance measures a stable mass $m_{\infty}$, corresponding to the average mass applied by the oscillatory acceleration of the needle $\ddot{y}_{\text {needle }}$.

millimeter. Both cameras have an electronic shutter as short as $300 \mathrm{~ns}$. Back illumination was provided by a light-emitting diode (LED) cold light source (SCHOTT KL 2500 LED) and a white diffuser in between.

\section{Needle displacement and force measurement}

The needle vertical displacement was characterized, varying the nominal frequency $f_{n}$ from 50 to $150 \mathrm{~Hz}$, with videos at $10000 \mathrm{fps}$ and a spatial resolution of 300 pixels per millimeter (see Appendix A). The force exerted by the solid needle injector into the agarose was measured by placing the injector and skin surrogate setup on a precision balance (Denver Instrument, APX-1000, $\Delta m=0.1 \mathrm{mg}$ ) [see Fig. 1(c)]. The injector exerts a normal force $F_{\text {needle }}$ on the agarose, recorded by the balance as an effective 
mass $m_{\mathrm{eff}}$

$$
F_{\text {needle }}=m_{\text {eff }}|\vec{g}| \text {, }
$$

where $|\vec{g}|$ is the gravitational acceleration.

When the solid needle starts moving, an initial peak mass $m_{\text {rupture }}$ is measured, corresponding to the agarose gel rupture as the needle penetrates the gel. Next, the measured mass reaches a stable value $m_{\infty}$, corresponding to the average effective mass caused by the oscillatory movement of the needle $y_{\text {needle. The effective }}$ mass values $m_{\text {rupture }}=0.34 \pm 0.04 \mathrm{gr}$ and $m_{\infty}=0.16 \pm 0.02 \mathrm{gr}$ were measured on agarose gels of $1 \%$ wt. at a needle displacement frequency of $f_{m}=70 \mathrm{~Hz}\left(f_{n}=50 \mathrm{~Hz}\right)$, with corresponding normal forces: $F_{\text {needle }}^{\text {rupture }}=3.3 \mathrm{mN}, F_{\text {needle }}^{\infty}=1.6 \mathrm{mN}$ (see comparison with the force exerted by liquid jets in Sec. V).

\section{B. Needle-free microjet injector}

A continuous wave $(\mathrm{CW})$ laser diode with a wavelength $\lambda=450 \mathrm{~nm}$ was focused at the bottom surface of a glass microfluidic device. In the first experiments, an aqueous solution of a molecular dye matching the laser wavelength partially fills the device. The liquid heats up above its boiling point in a few microseconds with an explosive phase transition resulting in a fast growing vapor bubble inside the microdevice. The bubble pushes the liquid forming a jet, which in turn can penetrate into an agarose gel located in front, as shown in Fig. 2. The microfluidic devices used were similar to those described elsewhere. ${ }^{28,29}$ Since the commercial inks used also absorb in the laser wavelength, a similar thermocavitation was observed and described in Sec. IV.

The microfluidic devices were designed and fabricated in glass substrates under cleanroom conditions. ${ }^{29}$ Each device has a fluidic chamber in which bubbles are created and is connected to a tapered channel with a $120 \mu \mathrm{m}$ diameter nozzle. Additional details of this setup are provided in Appendix B.

The laser diode is focused at the bottom of the device with a $10 \times$ microscope objective. The spot has an elliptical shape, with beam diameters $r_{x}=33 \mu \mathrm{m}$ and $r_{y}=6 \mu \mathrm{m}$ and variable power $\mathrm{P}=400-600 \mathrm{~mW}$. The range of powers was selected considering the energy required to create cavitation, based on the experimental

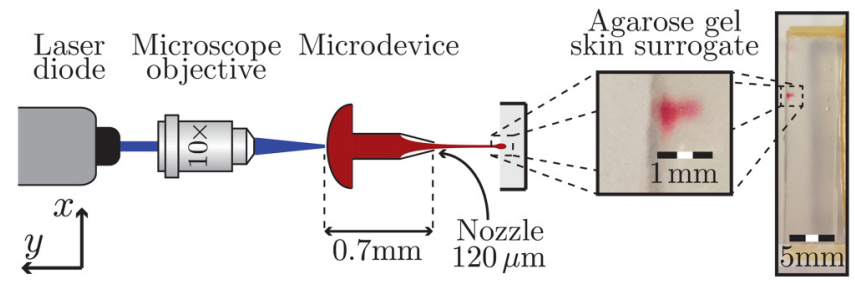

FIG. 2. Schematics of the needle-free microjet injector setup: A laser is focused at the bottom of a microfluidic device using a microscope objective. As a result, the bubble and jet are formed and are recorded using an ultrahighspeed camera. The liquid jet penetrates the skin surrogate located in front of it. The image and the zoom-in insets show the agarose gel holder and the injection print, respectively, using a red colored glycerol-water mixture at $10 \%$ wt. setup conditions. The transparent glass walls of a custom-made agarose holder permit the visualization of injection processes, as shown in the inset of Fig. 2. The agarose depth ( $y$-axis), width ( $x$-axis), and length ( $z$-axis) are fixed at $\sim 5 \mathrm{~mm}, 3 \mathrm{~mm}$, and $24 \mathrm{~mm}$, respectively.

The bubble growth, the liquid jet formation, and the penetration into agarose slabs were recorded at $\sim 400000 \mathrm{fps}$ using an ultrahigh-speed camera (Phantom v2640). The camera sensor is protected from the laser light using a colored glass filter centered at $\lambda=450 \mathrm{~nm}$. The camera has an electronic shutter as short as $150 \mathrm{~ns}$. As in the solid injector setup, the system is illuminated from the back using a LED cold light source (SCHOTT KL 2500 LED) and a white diffuser in between. It should be noted that the microscope objective used to focus the laser is not used in obtaining the images during injection.

\section{Inks' characterization and skin surrogate preparation}

Commercially used permanent make-up (PMU) inks are colloidal dispersions with flow-dependent viscoelastic properties. They are composed mainly by water and glycerol and extra additives such as surfactants, solvents, binders, and fillers. ${ }^{31,32}$ Pigments provide color, and due to insolubility in water, they guarantee the permanent character of the injected ink. Additives are used in order to avoid pigment sedimentation while storage and help the redispersion of the fluid after it is used. Microbiological contamination is common in tattoos due to the high content of water and organic substances present on inks. In order to avoid the contamination, preservatives are added to the mixture. Other impurities that can be found on tattoos are primary aromatic amines (PAA) and polycyclic aromatic hydrocarbons (PAH). ${ }^{6,7}$ In this study, we used two organic PMU inks: PMU-black (Amiea, Organic line, Deep Black, MT.Derm) and PMU-red (Amiea, Organic line, Cranberry, MT.Derm).

Since the actual composition of commercial inks is a trade-secret, the PMU inks mentioned above were compared with aqueous inks prepared by us, containing red dyed water and red dyed glycerin-water mixture at $10 \% \mathrm{wt}$. Adding glycerin changes the adhesion to the needle tip and the diffusion in the agarose is modified with respect to pure water. In order to maximize the absorbed energy by the liquid from the focused laser, in the needlefree microjet injector, the aqueous solutions are colored using a red dye (Direct Red 81, CAS No. 2610-11-9) diluted at $0.5 \%$ wt.

We performed rheology measurements of the liquid inks, specifically the viscosity $\eta$, varying the shear rate $\dot{\gamma}$ using a rheometer (Anton Paar MCR502) with a cone-plate geometry (with diameter $d=50 \mathrm{~mm}$ and cone angle $\alpha=1^{\circ}$ ). The red colored water and the glycerin-water mixture behave as Newtonian fluids with measured constant viscosity $\eta_{\text {water }}=0.9 \mathrm{mPa} \cdot \mathrm{s}$ and $\eta_{\text {glyc10\% }}=$ $1.2 \mathrm{mPa} \cdot \mathrm{s}$ (see Fig. 3). The PMU inks show a shear thinning behavior, i.e., the viscosity of the fluid decreases when the applied shear rate increases. The measured viscosity values at high shear rates, $\dot{\gamma} \gtrsim 100 \mathrm{~s}^{-1}$, for the PMU-black and PMU-red inks are $\eta_{\mathrm{PMU}-\text { black }} \sim 300 \mathrm{mPa} \cdot \mathrm{s}$ and $\eta_{\mathrm{PMU}-\mathrm{red}} \sim 2000 \mathrm{mPa} \cdot \mathrm{s}$, respectively. The larger viscosity value of PMU inks is provided by the high 


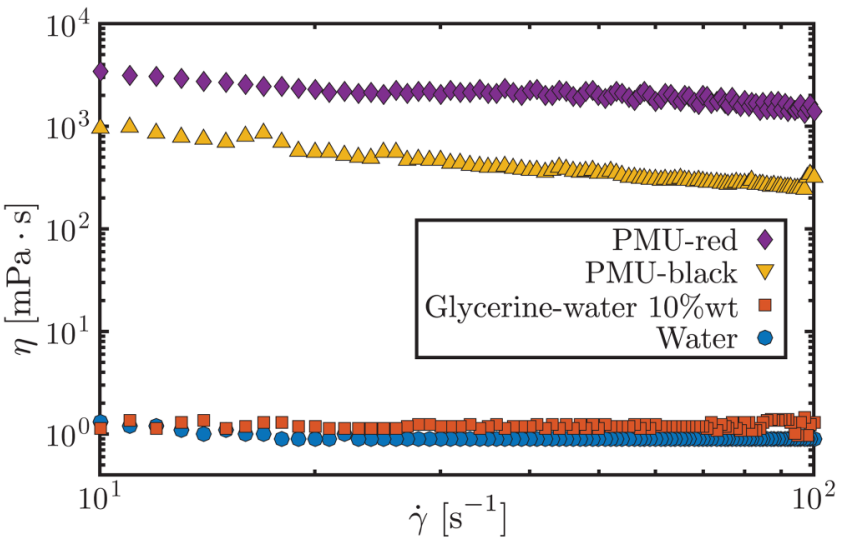

FIG. 3. The viscosity $\eta$ vs shear rate $\dot{\gamma}$ of the liquid inks: red dyed water (blue circles), red dyed glycerin-water $10 \%$ wt. (red squares), PMU-black ink (yellow triangles), and PMU-red ink (purple diamonds).

concentration of pigment particles, other additives not present in the colored water, and water-glycerin solutions.

Among the skin surrogates used to simulate the mechanical properties of soft tissues, agarose is one of the most popular due to its transparency, which allows the optical quantification of injections. ${ }^{24,29,33-36}$ The surrogates were prepared by diluting agarose powder (OmniPur agarose, CAS No. 9012-36-6), in deionized water, with an agarose concentration of $1 \%$ wt. The solution was heated up $45 \mathrm{~s}$ in microwave at full power. Once the phantoms were prepared, they were cooled down at room temperature for $5 \mathrm{~min}$ and stored at $4{ }^{\circ} \mathrm{C}$.

\section{SOLID NEEDLE INJECTION METHOD}

We studied the solid needle injector method in three stages: microindentation, stationary injection, and moving injection. Microindentation occurs when the needle pushes the skin surrogate down without causing the rupture of the surrogate. The stationary injection begins when the needle starts bringing ink into the surrogate, immediately after the rupture occurs, keeping the gel fixed with respect to the injector hand piece. Finally, the moving injection process corresponds to a scenario closer to real life injection conditions, in which the needle is moved perpendicular to the skin surface.

\section{A. Dynamic microindentation hardness test}

The interest to develop microindentation testing to characterize skin surrogate hydrogels has increased lately. ${ }^{37-42}$ Microindentation tests help in assessing the hardness of a material against deformation with low applied loads. We used a specific indentation testing configuration termed conical indenter (see Appendix C). The needle solid injector can be considered a dynamic microindentation measuring instrument with a conical indenter. The needle travels rapidly and does not allow the relaxation of the gel. Consequently, the measured force is the instantaneous force $F_{0}$ in relation to the needle displacement. The microindentation measurement is only valid before the rupture of the surface agarose gel, typically within one millisecond. Furthermore, the tip of the solid needle has a circular conical geometry, as shown in Fig. 12 in appendixes, with a height and a base radius of $h_{\text {tip }}=0.174 \mathrm{~mm}$ and $r_{\text {tip }}=0.068 \mathrm{~mm}$, respectively. The needle tip position $\delta$ and the gel surface deformation are obtained from experimental image sequences.

The gel deformation during the indentation process is plotted in Fig. 13(a), where the zero position is defined in the initial agarose gel surface plane, i.e., the surface before injection. We observed three stages in the surface deformation: capillarity, indentation, and injection. For a time $<2 \mathrm{~ms}$, when the needle is approaching, the first contact is with a water film in the surface of the gel, formed due to the environmental humidity and evaporation. At the contact point, a liquid bridge wetting the needle is created due to the capillary action, and the visualized deformation is negative $\delta<0$. The liquid bridge formation occurs too quickly to be captured in greater detail by our high-speed camera; however, this phenomenon plays an important role in, for example, nano- and microindentation and AFM microscopy. ${ }^{43,44} \mathrm{Next}$, when $y_{\text {needle }} \geq 0$ and $t \geq 0.2 \mathrm{~ms}$, the needle pushes the agarose gel down, starting the dynamic microindentation process. Finally, the force applied by the needle against the agarose is high enough to induce gel rupture, delivering ink effectively and starting the injection process.

The maximum deformation measured was $\delta_{\max }=0.1062 \mathrm{~mm}$, which corresponds to the applied force $F_{\text {rupture }}=3.3 \mathrm{mN}$. We compare this force with $F_{0}$ as $F_{\text {rupture }}=3.3 \mathrm{mN}=$ $4 G \delta_{\max } r_{\max }=F_{0}$, where $r_{\max }=r_{\text {tip }} \delta_{\max } / h_{\text {tip }}=0.042 \mathrm{~mm}$. We can calculate the shear modulus $G$ of the skin surrogate, which represents the hardness, rigidity, or stiffness of the material, obtaining $G=185 \mathrm{mN} / \mathrm{mm}^{2}=185 \mathrm{kPa}$. This value matches the shear modulus range, $\sim[30-3000] \mathrm{kPa}$, reported in the literature for the agarose concentrations used. ${ }^{45,46}$

\section{B. Stationary injection}

Stationary injections were performed to understand the delivery process without the influence of specific factors, such as the needle injection angle, and translational speed with respect to the skin surrogate. The injection process in the case of a new needle holder loaded with PMU-black ink is shown in Fig. 4(a) (Multimedia view). We observed that it takes over 50 injections for the ink adhered to the needle surface to slide down and initiate the delivery into the agarose; this instant is considered $t=0$. After that, another 50 injections are needed to make a spot-width equivalent to the needle diameter of $0.4 \mathrm{~mm}$. Finally, around 100 injections later, the injection width reaches a threshold value of $\sim 0.8 \mathrm{~mm}$. This plot shows that a unique injection is not always enough to deliver a dose equivalent to the needle volume. Figure 4(b) (Multimedia view) shows a high-resolution image sequence of the agarose gel after four injections without ink. The deformation of the agarose gel surface after only four injections is clearly visible. Also, the gel is internally damaged, and a longitudinal hole of a diameter $\sim 0.1 \mathrm{~mm}$ remains after one injection. After the second injection, a darker region inside the hole was observed, corresponding to a small water drop that came out of the agarose gel. 

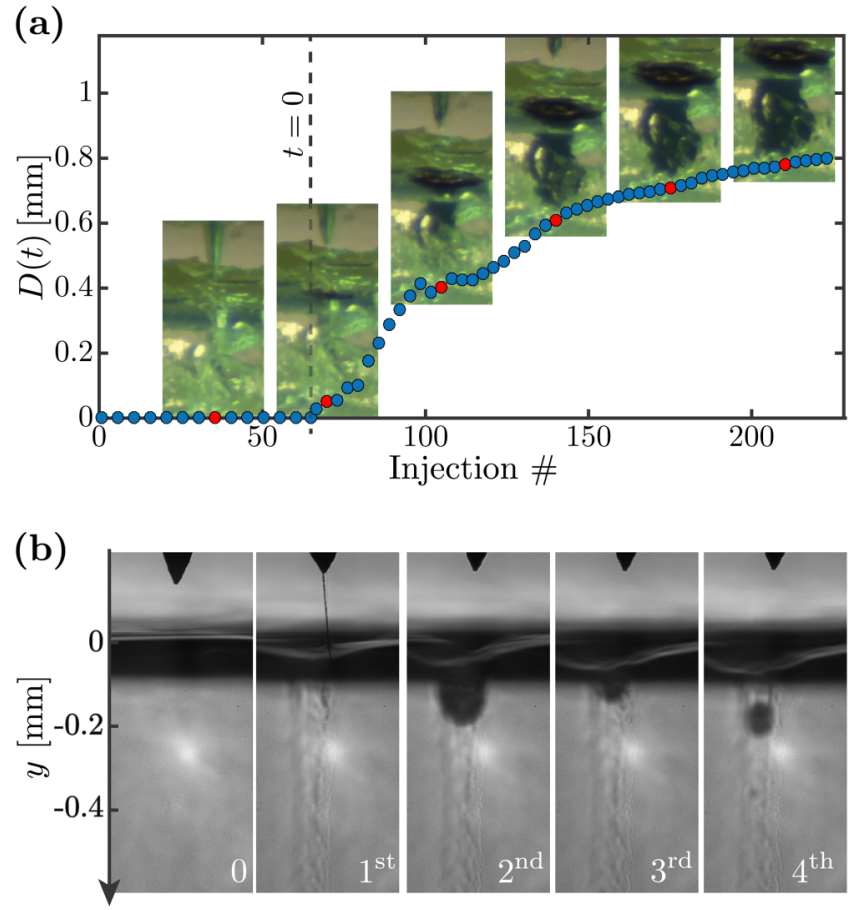

FIG. 4. (a) Injected-ink, $D(t)$, vs injection number. A clean needle holder is loaded with PMU-black ink delivering ink into the agarose after $\sim 60$ injections; this moment is considered $t=0$ (dashed line). Around 100 injections later, the injection width reaches a threshold value $\sim 0.8 \mathrm{~mm}$. Every image shows the injection at the time corresponding to the red point. (b) High-resolution images show the skin surrogate agarose gel after 4 injections. The continuous deformation of the agarose gel surface is observed after each frame. A longitudinal hole of a diameter $\sim 0.1 \mathrm{~mm}$ remains in the agarose gel after every injection. Multimedia views: https://doi.org/10.1063/1.5074176.1; https://doi.org/10.1063/1. 5074176.2

The injection process, from $t=0$, is compared for three of the inks: PMU-black ink, glycerin-water mixture, and water. We calculated the ratio between the injection depth $L(t)$ and the immersed needle length $L_{\text {needle }}$ and between the injection width $D(t)$ and the immersed needle diameter $D_{\text {needle }}$ [see Figs. 5(a) and 5(b)]. The total needle length is around $2 \mathrm{~mm}$, but the total immersed needle into the agarose can vary in dependence of the initial distance between the needle tip and the agarose surface, mainly due to the agarose surface unevenness. We observe that $L(t) / L_{\text {needle }}$ increases slower proportionally to the ink viscosity, but after $\sim 40$ injections, they all reach a threshold value $L / L_{\text {needle }}=1$.

In the case of $D(t) / D_{\text {needle, the spreading behavior is the oppo- }}$ site; for the PMU ink, the change from zero to one is almost instantaneous and continues to increase up to $D / D_{\text {needle }}=2$ in around 100 injections. For the aqueous solutions, $D(t) / D_{\text {Needle }}$ saturates very quickly to 1 , and the water and glycerin-water curves are almost indistinguishable [yellow diamonds and green squares in Fig. 5(b)].
Assuming a circular conical injection shape, we estimate the injected volume as

$$
V(t)=\frac{1}{3} \pi\left(\frac{D(t)}{2}\right)^{2} L(t)
$$

and plotted in Fig. 5(c). The image insets show the delivered volume at the same time $\sim 1 \mathrm{~ms}$ or the same injection number $\sim 75$. The $V(t)$ for the PMU ink increases at least twice faster than for the aqueous solutions, with volumes of $\sim 80 \mathrm{nl}$ and $\sim 20 \mathrm{nl}$, respectively, as expected from the $L(t) / L_{\text {needle }}$ and $D(t) / D_{\text {needle }}$ plots.

The large differences in behavior are highly correlated with the differences in viscosity. For the solid needle used, with diameter $D_{\text {needle }}=0.4 \mathrm{~mm}$ and an injection frequency and amplitude $f_{m}=74 \mathrm{~Hz}, a_{m}=1 \mathrm{~mm}$, the shear rate of the ink film wetting the needle is

$$
\dot{\gamma}=\frac{v_{\text {needle }}}{D_{\text {needle }}}=\frac{a_{m} 2 \pi f_{m}}{D_{\text {needle }}}=1000 \frac{1}{s} .
$$

This means that the viscosities of aqueous and the PMU inks differ by two orders of magnitude $(1 \mathrm{mPa} \cdot \mathrm{s}$ and $300 \mathrm{mPa} \cdot \mathrm{s})$. The PMU ink adheres better to the needle, making it necessary to clean the needle in between experiments to prevent ink agglomeration. Moreover, the images show that the residual-not injected-ink accumulates in the surface, making the solid needle injection method highly inefficient for low viscous inks. Furthermore, there is a clear difference in the observed liquid adhesion onto the needle when the viscosity increases from $\eta_{\text {water }}=0.9 \mathrm{mPa} \cdot \mathrm{s}$ to $\eta_{\text {glyc } 10 \%}=1.2 \mathrm{mPa} \cdot \mathrm{s}$. Figure 5 (c) show this difference, where the amount of ink remaining in the agarose surface is considerably higher for pure water than glycerin-water mixture.

\section{Moving injection}

A qualitative characterization of conventional tattoo injection processes was performed by moving the translation stage at $2 \mathrm{~mm} / \mathrm{s}$ orthogonal to the hand-piece. A total of 50 injections, corresponding to $t \sim 700 \mathrm{~ms}$, were studied. Figure 6 (Multimedia view) shows a characteristic image sequence of the injection processes with different inks: (a) PMU-black ink, (b) glycerin-water mixture, and (c) water. That PMU ink has a much smaller spreading into the agarose gel, which we attribute to the several ingredients providing cohesion (not present in glycerin-water mixture and water). In the case of the PMU inks, the surrogate saturation is faster and the ink oozes out on top of the agarose surface. This behavior is expected because the agarose gel is mainly composed of water, therefore the larger spread of the in-house prepared aqueous inks. Additionally, a residual volume of PMU ink remains adhered to the needle during the whole injection process. Pigment particles provide the higher viscosity and density of PMU inks, higher adhesion to the needle, and limit the spreading into the agarose. The tailored properties of PMU ink that facilitate the drying process in tattooing practice cause agglomeration and particle adhesion to surfaces further discussed in Sec. II C. 

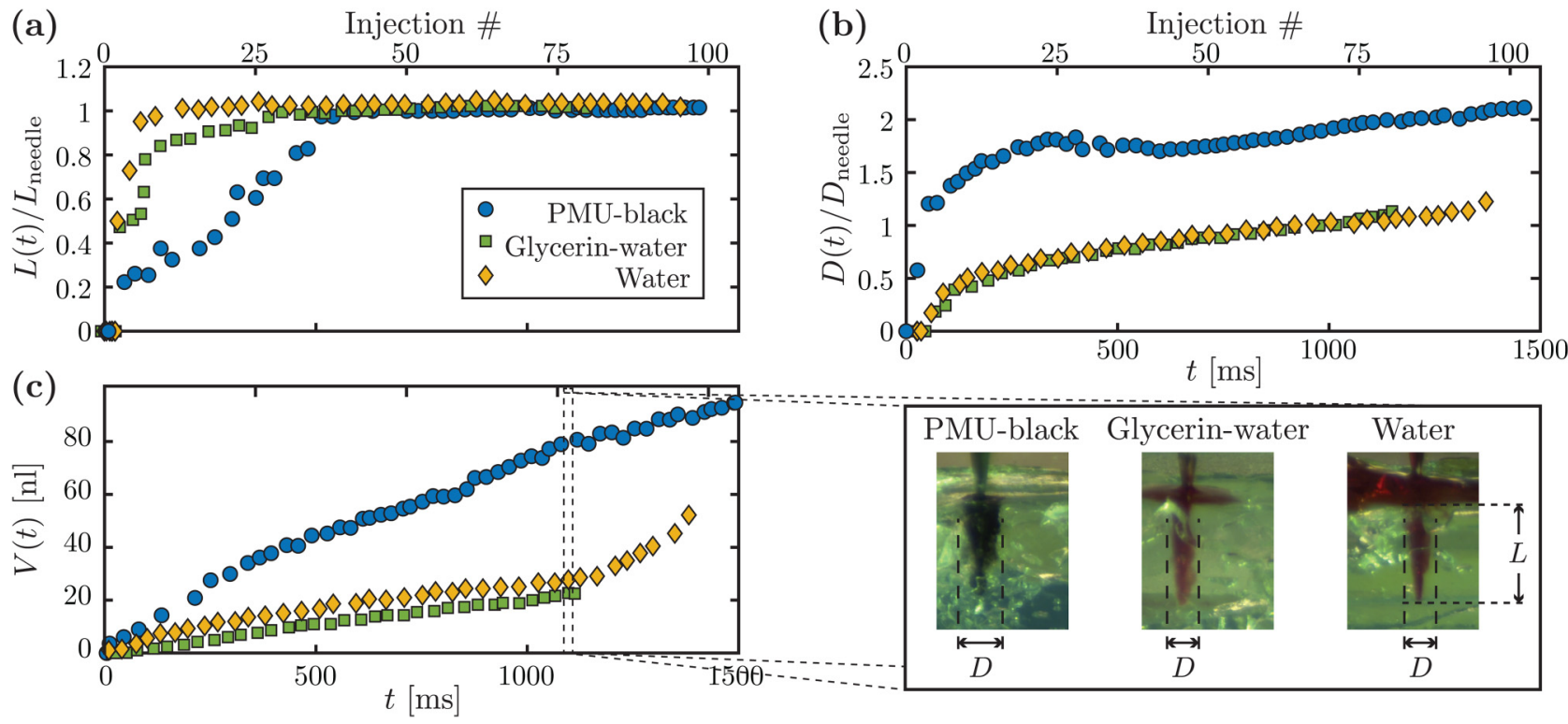

FIG. 5. (a) Injection depth and immersed needle length ratio $L(t) / L_{\text {needle, }}$ (b) injection width and immersed needle diameter ratio $D(t) / D_{\text {needle, }}$, and $(\mathrm{c})$ delivered volume $V(t)$ for three inks: PMU-black ink, glycerin-water mixture, and water. The insets show the delivered dose at the same time $\sim 1 \mathrm{~ms}$ and/or the same injection number $\sim 75$. The efficiency in volume delivery of PMU ink corresponds to the specific tailored properties for injection not present in the other formulations.

(a)

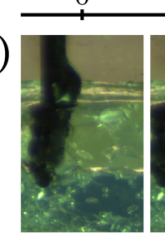

10

Injection \#
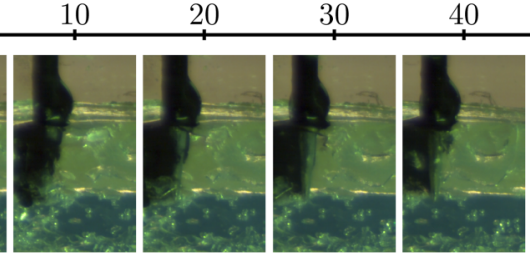

50

(b)
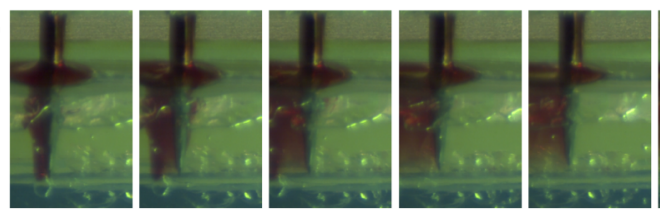

(c)
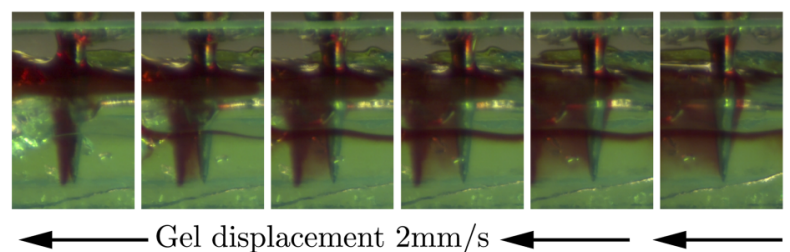

FIG. 6. Image sequences of the moving injection process for three inks: (a) PMU-black ink, (b) glycerin-water mixture, and (c) water. The solid needle injector is initiated and the agarose gel is moving at $2 \mathrm{~mm} / \mathrm{s}$, from right to left. The spreading of the ink into the agarose is faster and wider for aqueous solutions than the PMU ink, which has a viscosity 200 times higher. Multimedia views: https://doi.org/10.1063/1.5074176.3; https://doi.org/10.1063/1.5074176.4; https:// doi.org/10.1063/1.5074176.5
Photographs of the injected skin surrogate were taken immediately after the moving injection experiment using a compact digital camera (Nikon Coolpix A100). The agarose gel was cleaned before the imaging with a professional cleansing tonic (LaBina Aloe Vera tonic, PERMANENT-Line), in order to observe the postinjection result at the front and bottom, as shown in Fig. 7 for PMU-red ink, PMU-black ink, glycerin-water mixture, and water. For the PMU inks, a well defined path is visible in the front and bottom views. Due to its fast diffusion, the path made with glycerin-dyed water solutions is no longer visible. However, we observed that the glycerin-water mixture presents less spread than pure water [see Fig. 6 (Multimedia view)], in agreement with our observations in Sec. III B. The bottom view shows an asymmetrical spread around the needle, with a pattern depending on the microcharacteristics of the agarose gel, i.e., its porosity and other inhomogeneities.

Figure $7(\mathrm{e})$ shows the intensity of the injection path, as observed in the mirror, and averaged in the $x$ direction, with corresponding zoom-in at the mirrors on the right. Two well defined peaks for the PMU inks are observed in the plot. The PMU-red peak is wider than the PMU-black, because the red ink is one order of magnitude more viscous; hence, the ink attaches better to the needle. The aqueous inks spread completely in the agarose, showing a homogeneous average intensity. However, the glycerinwater mixture has a higher intensity, indicating that more ink has been delivered into the agarose.

\section{NEEDLE-FREE INJECTION METHOD}

The needle-free injector creates liquid microjets with a tip diameter $D_{\text {jet }} \sim 50 \mu \mathrm{m}$ and total ejected volumes of ca. $50 \mathrm{nl}$. 

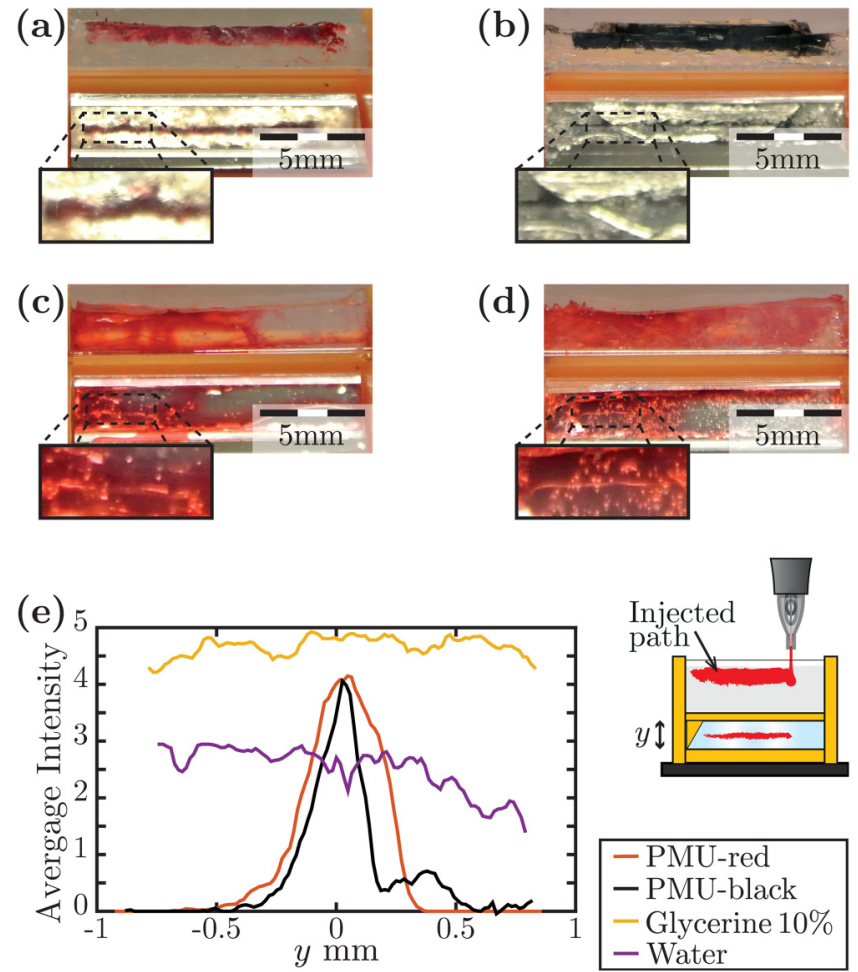

FIG. 7. Side and bottom view of postinjection skin surrogate for (a) PMU-red, (b) PMU-black, (c) glycerin-water mixture, and (d) water inks. The insets show a zoom-in of the injection path observed in the mirror (bottom view). (e) The average intensity of the injected path observed in the mirror is plotted, next to it, a schematic of the skin surrogate postinjection.

Around $100 \%$ of the liquid is delivered into the agarose for jet speeds larger than $\sim 40 \mathrm{~m} / \mathrm{s}$, with no splash-back of liquid due to the sufficient kinetic energy of the jets. Figure 8(a) (Multimedia view) shows image sequences of two successive water jets entering the agarose at the same point, both with a speed of $40 \mathrm{~m} / \mathrm{s}$ before impact. The depths reached by both injections are plotted against time in Fig. 8(b) (Multimedia view). In the first injection, the jet speed drops to $16 \mathrm{~m} / \mathrm{s}$, with a decrease of $85 \%$ in the jet kinetic energy, and penetrate $1 \mathrm{~mm}$ into the agarose. The first jet opens a hole into the skin surrogate, and subsequent jets follows through into a micrometric longitudinal orifice. Therefore, the second microjet terminal velocity reaches $20 \mathrm{~m} / \mathrm{s}$, with a kinetic energy decrease of $75 \%$, penetrating $40 \%$ deeper into the agarose.

Experiments with glycerin-water mixture were performed in order to compare the jet injection process with the solid needle injection method (Sec. III). The shear rate experienced by the liquid jets is at least two orders of magnitude higher than that provided by the solid needle injector. An image sequence of a single injection with microjet speed $v_{\text {jet }} \sim 25 \mathrm{~m} / \mathrm{s}, \dot{\gamma}=5 \times 10^{5} 1 / \mathrm{s}$, for pure water and glycerin-water mixture is shown in Fig. 9(a). The differences observed in the agarose color and texture are an optical effect due to small illumination differences. It can be observed that,
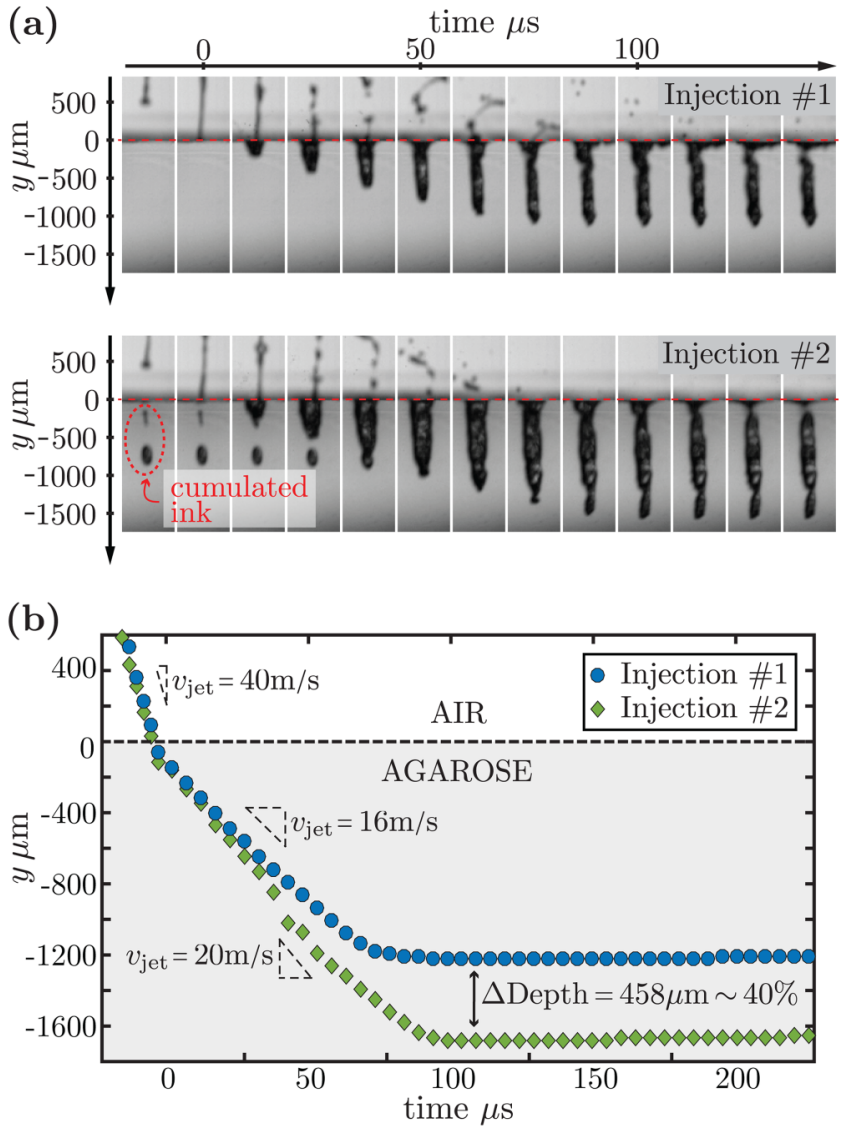

FIG. 8. (a) Two consecutive injections for pure water, with a jet speed of $40 \mathrm{~m} / \mathrm{s}$. The red-dashed line corresponds to the agarose gel surface. The first jet opens a hole into the skin surrogate, and the cumulated ink is visible into the gel. With the second injection, the total injected volume and depth increase. (b) Penetration depth vs time for the two continuous injections. Before the microjet reaches the agarose surface, both jets have a speed of $40 \mathrm{~m} / \mathrm{s}$; with the impact, the first injection drops its speed to $16 \mathrm{~m} / \mathrm{s}$, while the second injection to $20 \mathrm{~m} / \mathrm{s}$, penetrating around $40 \%$ deeper into the agarose. Multimedia views: https://doi.org/10.1063/1.5074176.6; https://doi.org/10.1063/1.5074176.7

despite having the same initial jet velocities, water jets penetrate deeper than glycerin jets. The corresponding complete penetration events are plotted in Fig. 9(b).

We observed that inertial effects are more relevant in the early stages of jetting and penetration, while viscous dissipation gains prominence toward the end-point injection. The penetration speed of both jets right after entering the agarose drops to a comparable speed, $8 \mathrm{~m} / \mathrm{s}$ and $7 \mathrm{~m} / \mathrm{s}$, respectively. Afterwards, during jet deceleration, water jets penetrate much deeper than glycerin-water jets ( $120 \%$ increase) due to the differences in the liquid viscosities of $25 \%\left(\eta_{\text {water }}=0.9 \mathrm{mPa} \cdot \mathrm{s}\right.$ and $\left.\eta_{\text {glyc } 10 \%}=1.2 \mathrm{mPa} \cdot \mathrm{s}\right)$. Though not visible in these figures, water jets spread (laterally) faster into the agarose gel, while the spread area of glycerin jets remains almost the same after each injection. 
(a)
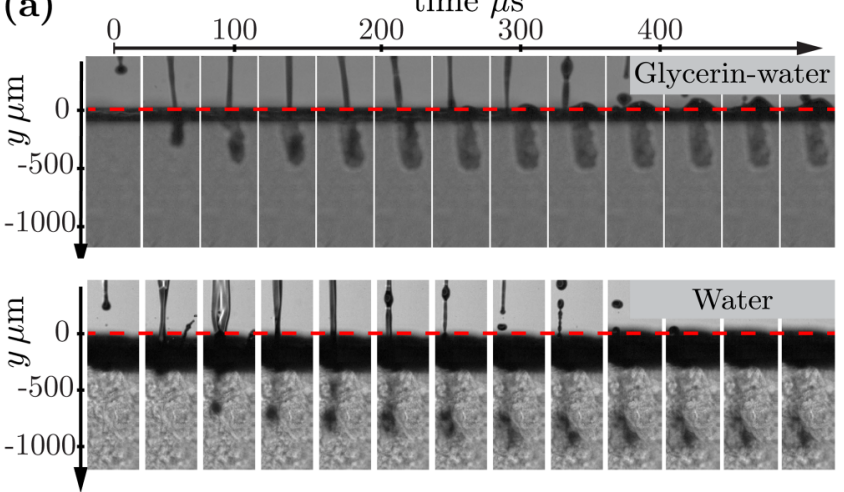

(b)

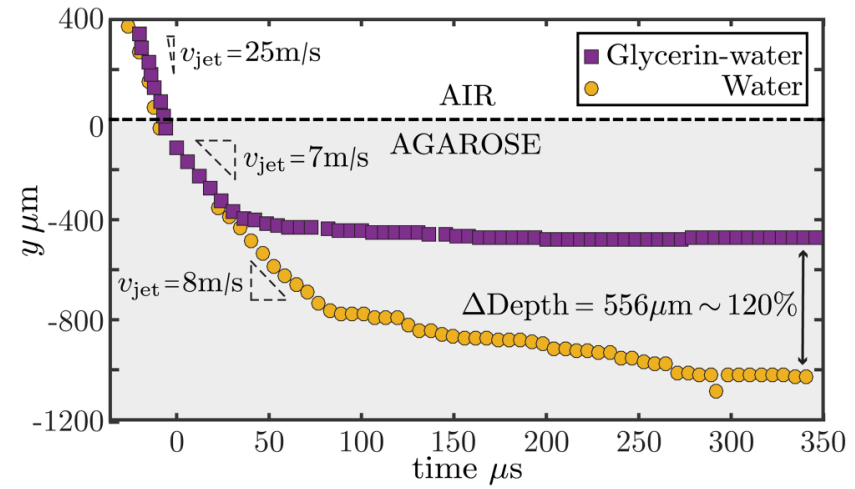

FIG. 9. (a) Image sequence of a single injection for pure water and glycerinwater mixture. In both cases, the microjet speed is $v_{\text {jet }} \sim 25 \mathrm{~m} / \mathrm{s}$ and the skin surrogate is agarose gel $1 \%$ wt. of concentration. (b) Penetration depth vs time for the two injections. The pure water ink is able to penetrate $120 \%$ deeper than the glycerin-water mixture.

In contrast with the solid needle injector, no damage was observed on the agarose surface after multiple injections recorded with an image resolution of 150 pixels per millimeter. Moreover, the agarose shows a capacity of self-recovery as the path created by the microjet closes and encapsulates the injected ink; see Fig. 8(a) (Multimedia view, first image, second injection).

The use of PMU inks in the needle-free microjet injector was an experimental challenge. First, visualization is limited because of light absorption by the pigments that impeded the observation of thermocavitation. Second, after one or two laser shots, the residual ink and particles agglomerate in an unforeseeable manner, limiting the reproducibility of the experiments (see Fig. 10).

\section{COMPARISON OF INJECTION METHODS}

The fact that no solid object is needed to rupture the surrogate with the needle-free microjet injector is a clear advantage in practical terms. To make a fair comparison, the agarose gel surface rupture and damage, the spreading of the ink, among other
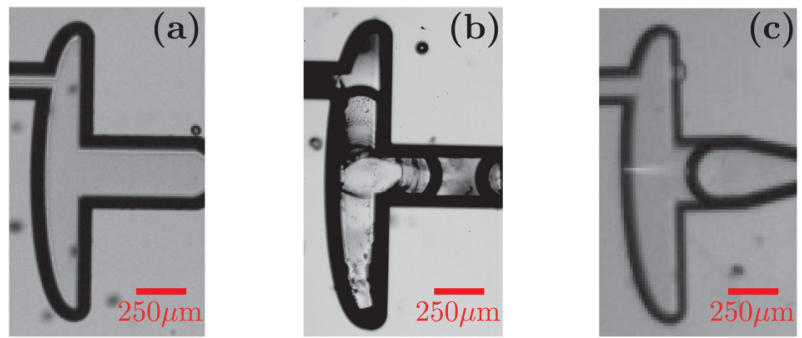

FIG. 10. Image of microdevices before being used (a), after a single laser shot using PMU-black ink (b), and red colored water (c). Notice the difference between residual particles adhered to the inner walls of (b) and that the channel in (c) was refilled automatically without residues.

observations are detailed in this section. The values calculated for all inks and both injection methods are presented in Table I, using the results in Fig. 5, for the solid needle, and Figs. 8 and 9, for the needle-free microjet.

The electric energy supplied to the injectors is calculated as $E=P \times t$. The solid needle injector input power is $P=7 \mathrm{~W}$, and the time of a single injection is $t=1 / 70 \mathrm{~s}$, with a consumed energy $E=100 \mathrm{~mJ}$. The needle-free microjet injector input power corresponds to the laser power $P_{\text {laser }}=0.5 \mathrm{~W}$ and the corresponding time needed for the liquid to cavitate $t=1 \mathrm{~ms}$, corresponding to $E=5 \mathrm{~mJ}$. The kinetic energy transferred from the liquid to the skin is calculated as $K=1 / 2 m v^{2}$, where the speed for the solid needle is $v_{\mathrm{SN}}=a_{m} 2 \pi f_{m}$ and for the jet is $v_{\text {jet }}$. Therefore, the injection efficiency in terms of energy per injection is calculated as $\epsilon_{\text {energy }}=K / E \times 100 \%$. We have calculated that the needle-free microjet injector has a $\epsilon_{\text {energy }}$ three orders of magnitude higher than the solid injector (Table I, sixth column).

The liquid volume deposited by the solid needle injector in each injection was estimated as the thickness of the liquid film around the needle tip,

$$
\delta=c\left(\frac{\eta v}{\rho g}\right)^{1 / 2}
$$

where $\eta$ is the liquid viscosity, $\rho$ the liquid density, $v$ the flow velocity, and $g$ the gravitational acceleration. This equation assumes that the flow velocity corresponds to the needle velocity and that the surface tension role is negligible. ${ }^{47,48}$ The constant $c$ is taken as 0.8 , which is a standard value for most Newtonian fluids. The ink volume around the needle is estimated as the volume of a hollow cylinder with the inner radius as the needle radius $r_{\text {inner }}=$ $D_{\text {needle }} / 2$ and outer radius $r_{\text {outer }}=D_{\text {needle }} / 2+\delta$.

With the injection efficiency in terms of volume injected per injection event, we calculate it as the ratio between the deposited (solid needle) or ejected (needle-free microjet injector) volume $V_{0}$ and the volume remaining inside the agarose gel. For the former case, this is defined as the moment in which the solid needle retracts and the latter is after the hole in the agarose closes. This efficiency is represented as 
TABLE I. Comparison between the solid needle and needle-free microjet methods. $S$ is the strength ratio between the injection pressure $p$ and the skin surrogate shear modulus $G=185 \mathrm{kPa}$ obtained in Sec. III A. In the case of the solid needle, the penetration depth $L$, width $D$, and volume $V$ are quantified for a single injection and for the end point.

\begin{tabular}{|c|c|c|c|c|c|c|c|c|c|c|c|}
\hline \multirow[b]{2}{*}{ Method } & \multirow[b]{2}{*}{ Ink } & \multirow[b]{2}{*}{$p(\mathrm{kPa})$} & \multirow[b]{2}{*}{$S=p / G$} & \multirow[b]{2}{*}{$K(\mu \mathrm{J})$} & \multirow[b]{2}{*}{$\boldsymbol{\epsilon}_{\text {energy \% }}$} & \multirow[b]{2}{*}{$V_{0}(\mathrm{nl})$} & \multicolumn{3}{|c|}{ \#1 injection } & \multicolumn{2}{|c|}{ End point } \\
\hline & & & & & & & $L(\mathbf{m m})$ & $D(\mathrm{~mm})$ & $\overline{\epsilon_{\mathrm{vol}} \%}$ & $L(\mathbf{m m})$ & $D(\mathrm{~mm})$ \\
\hline \multirow[t]{3}{*}{ Solid needle } & PMU-black & 2400 & 13 & 0.042 & $4.2 \times 10^{-5}$ & 424 & 0.047 & 0.129 & 0.005 & $\sum^{50}=1.5$ & 0.8 \\
\hline & Glycerin-water & & & 0.033 & $3.3 \times 10^{-5}$ & 338 & 0.154 & 0.021 & 0.004 & $i=1$ & 0.4 \\
\hline & Water & & & 0.027 & $2.7 \times 10^{-5}$ & 283 & 0.197 & 0.019 & 0.004 & & 0.4 \\
\hline \multirow[t]{2}{*}{ Needle-free microjet } & Glycerin-water & 313 & 1.69 & 2.85 & $5.7 \times 10^{-2}$ & 8.9 & 0.450 & 0.192 & 88.1 & $\ldots$ & $\ldots$ \\
\hline & Water & 800 & 4.32 & 16.16 & 8.1 & 20.2 & 1.2 & 0.205 & 75.3 & $\sum_{i=1}^{2}=1.65$ & 0.205 \\
\hline
\end{tabular}

$\epsilon_{\mathrm{vol}}=V_{\mathrm{inj}} / V_{0} \times 100 \%$. For the solid needle, the efficiency increases with each new injection because of the remaining ink adhered to the needle from prior injections. There is, however, excess of liquid on the top of the agarose gel that does not penetrate at all and contributes to the well known ink loss of $50 \%$ in tattooing and permanent makeup procedures. ${ }^{49,50}$ To our surprise, the $\epsilon_{\mathrm{vol}}$ of the needle-free microjet injector is five orders of magnitude larger for the solid needle.

In order to quantify the skin rupture and penetration characteristics, we compare the local stress induced by the solid needle and the jet impact, with a material-dependent critical local stress as proposed before. ${ }^{19}$ We define a new quantity, the penetration strength $S$, as the ratio between the injection pressure $p$ and the skin surrogate shear modulus $G=185 \mathrm{kPa}$ calculated in Sec. III A. In the case of the solid needle, the injection pressure was calculated as the ratio between the rupture force $F_{\text {rupture }}$ and the needle crosssectional area,

$$
p_{\text {needle }}^{\# 1}=\frac{F_{\text {rupture }}}{A_{\text {needle }}}=\frac{F_{\text {rupture }}}{\pi r_{\max }^{2}},
$$

where $r_{\max }=0.042 \mathrm{~mm}$ is the radius of the immersed needle volume at the maximum deformation of the skin surrogate (see Sec. III A). The pressure exerted by the jet onto the skin is $p_{\text {jet }}=\frac{1}{2} \rho v_{\text {jet }}^{2}$, where $\rho$ is the density of the liquid and $v_{\text {jet }}$ the jet speed. $^{19}$

The penetration depth $L$ and diameter $D$ in the latest stages or end-point of injection-after 50 injections for the solid needle and after two injections for the needle-free injectors-show two interesting results. First, that with only two jets is possible to reach the same depth as with solid needles. Second, that the injection resolution in spreading value $(D)$, which corresponds to the practical level of detail in tattooing, is half for the jet injections. The current experimental setup for jet injections did not allow the same reproducibility of conditions to reach larger number of injections. Contrary to the solid needle injections, each new jet carries a slightly higher velocity because of the reduction in liquid inside the microfluidic chamber. In future studies, new microfluidic device designs should ensure that all jets in a sequence are ejected with the same velocity and total volume.

\section{CONCLUSIONS}

We consider that this study will advance the knowledge of injection processes into soft substrates based on solid needles and needle-free microjet injectors. Our novel needle-free microjet injector employing thermocavitation is still in early development phases; however, the results indicate that the injection outcomes are superior to solid needle injectors in several aspects. Further work is required, particularly aimed at increasing the repeatability, frequency of injections, and overcoming challenges related to the use of commercial inks with unknown ingredients. An interesting separate study would be to test the injectors compared in this work on real skin samples, both in vitro and in vivo. Our results indicate the following:

- The power consumption of needle-free jet injections, $0.5 \mathrm{~W}$, is an order of magnitude lower than the solid injector $(7 \mathrm{~W})$. This has practical relevance in applications where the portability of injector devices is limited by the need of incorporating heavy batteries.

- The calculated penetration strength $S$ reflects the proportional final damaged state of the skin surrogate. Values of $S$ slightly above unity (jet injections) seem to correspond to an effective penetration with minimal damage, in contrast with irreversible skin surrogate deformations when $S \sim 10$ (solid needle).

- The damage to the gel during the moving injections indicates that needle-free microjet injectors could have a less negative effect injecting into skin than solid needles. In real-life conditions, the displacement velocity and pressure applied with a hand-piece can fluctuate and damage the skin due to the solid needle hardness.

- The injection spreading increases with decreasing ink viscosity. However, the volume and energy efficiencies seem to increase proportionally to the viscosity of inks.

- The injection efficiencies, $\epsilon_{\text {energy }}$ and $\epsilon_{\text {vol }}$, are higher for the needle-free microjet injector, with comparable end-point injections. The needle-free microjet injector in a single injection reaches penetration depths and widths comparable to $\sim 50$ injections with a solid needle. Even though multiple injections were not possible with the current needle-free microjet injector, the higher efficiency values demonstrate the superiority over the 
older solid needle method. For needle-free microjet injectors to compete with established injection methods in real-life scenarios, however, future designs should give multiple and reproducible injections, e.g., with multiple nozzles or microfluidic geometries ensuring self-refill of ink after each jet. To avoid the problems of particle occlusion of the microchannels, separate studies could focus on having a special surface functionalization of the inner walls of the channel. Similarly, inks could be developed with specific particle size distributions and surface properties as to avoid clogging.

In short, needle-free microjet injections hold potential to mitigate health problems associated with needle-based injections and avoid technical limitations of solid needle injections in medical treatments, e.g., vaccines, scar camouflaging, alopecia, etc. The reduction of environmental contamination with needle-free injections could be immense, while benefiting millions of people using needles for medical and cosmetic uses. With a long list of challenges ahead, we believe there will be a time, not far from now, when needle-free microjet injectors will be reliable, safer, and efficient liquid delivery platforms, and where needles will not be that much needed.

\section{ACKNOWLEDGMENTS}

We would like to thank Stefan Schlautmann and Frans Segerink for their technical support during fabrication and optical setup construction. We also thank Edgerton's center for the use of the Phantom high-speed camera and illumination. The material support of PERMANENT-Line GmbH \& Co. KG and MT.Derm is kindly acknowledged, as well as the practical and theoretical training offered by PERMANENT-Line GmbH \& Co. KG. D.F.R. acknowledges the recognition from the Royal Dutch Society of Sciences (KHMW) that granted the Pieter Langerhuizen Lambertuszoon Fonds, 2016.

\section{APPENDIX A: NEEDLE DISPLACEMENT}

The needle tip position was obtained and plotted with respect to time, as shown in Fig. 11(a). During the first $\sim 0.15 \mathrm{~s}$, the needle vertical displacement $y_{\text {needle }}$ is the same for every nominal frequency. A slow oscillation is followed by a cyclical displacement with constant frequency $f_{0}=122 \pm 1 \mathrm{~Hz}$ and measured amplitude $a_{m}=1.018 \pm 0.006 \mathrm{~mm}$. After this time, $t \gtrsim 0.15 \mathrm{~s}$, the measured frequency $f_{m}$ reaches a stable value directly correlated to $f_{n}$. In Fig. 11(b), we plot the power spectral density of $y_{\text {needle }}$ at $t>0.15 \mathrm{~s}$ for the 3 cases shown in Fig. 11(a); the position of the first peak corresponds to the measured frequency $f_{m}$. In Fig. 11(c), we plot $f_{m}$ vs $f_{n}$ for all the experiments giving a linear relation $f_{m}=0.5 f_{n}+42$.

\section{APPENDIX B: MICROFLUIDIC DEVICE DETAILS}

Boroflat glass wafers were micromachined using wet-etching in hydrogen fluoride solutions. The micromachined design of the channel on two wafers defines the chamber that contains the liquid after attaching them together with anodic bonding. The bonded wafers were diced into individual microfluidic devices of
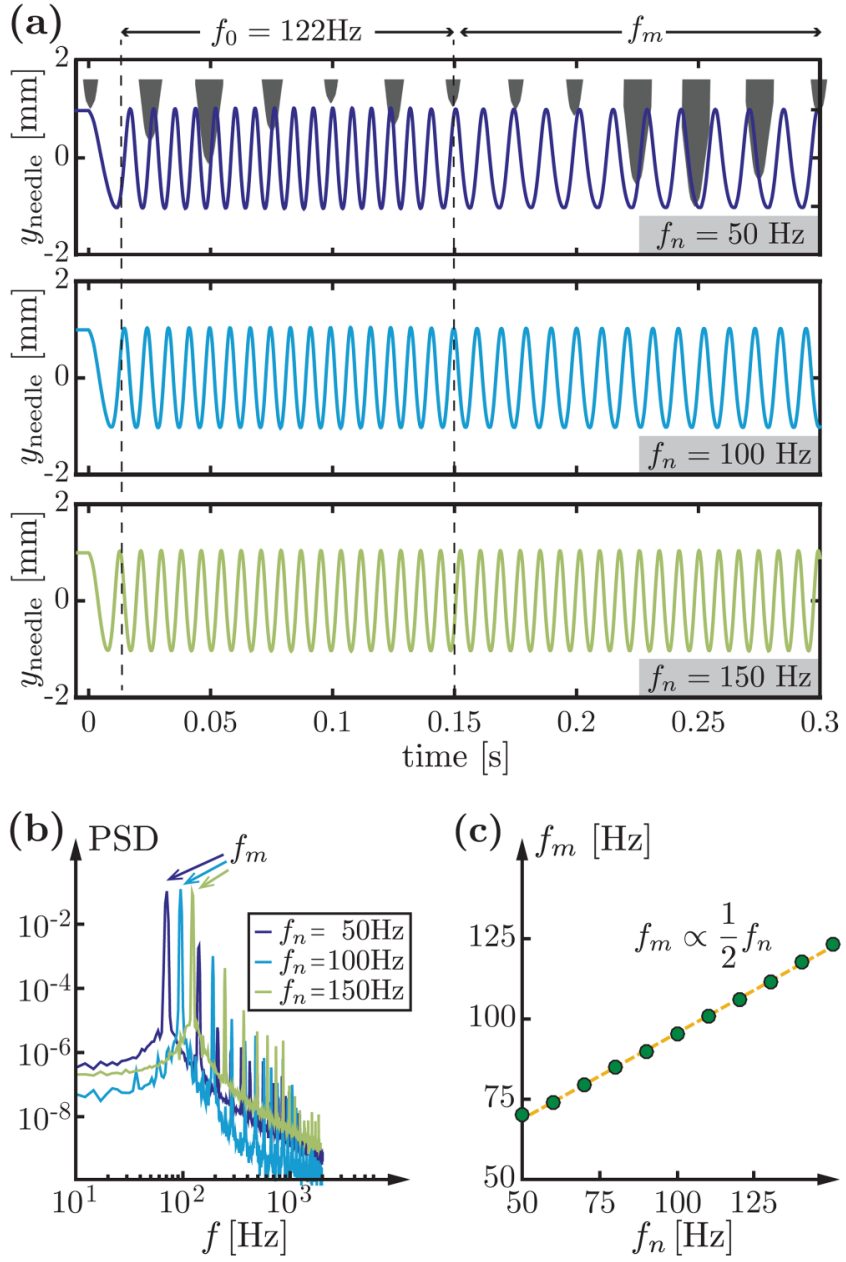

FIG. 11. (a) Vertical position of the needle tip vs time, for nominal frequencies $f_{n}=50 \mathrm{~Hz}, 70 \mathrm{~Hz}$, and $90 \mathrm{~Hz}$. During the first $\sim 0.15 \mathrm{~s}$, the needle oscillations are the same in all cases. A slow oscillation is followed by a frequency $f_{0} \sim e q 122 \mathrm{~Hz}$, after which a stable measured frequency $f_{m}$ is reached in correspondence with the different nominal frequencies. (b) The power spectral density of the needle $y$-position for $t 0.15 \mathrm{~s}$ is plotted, for nominal frequencies $f_{n}=50 \mathrm{~Hz}, 100 \mathrm{~Hz}$, and $150 \mathrm{~Hz}$. The first peak corresponds to the measured frequency $f_{m}$. (c) The measured frequency $f_{m}$ vs the nominal frequency $f_{n}$ is also plotted. The fitted curve is represented by the dashed line and shows a linear dependence $f_{m} \propto 1 / 2 f_{m}$.

$10 \times 8 \mathrm{~mm}^{2}$. The channel inlet $(400 \mu \mathrm{m}$ depth) connects to a circular container $(100 \mu \mathrm{m}$ depth), followed by a meandering channel as a fluid resistor. The bubbles are created in a chamber $(100 \mu \mathrm{m}$ depth), and the liquid is rushed onto an exit channel with length $500 \mu \mathrm{m}$. The liquid inlet was connected to a $250 \mu$ l Hamilton $^{\circ}$ GASTIGHT $^{\varpi}$ syringe through a glass capillary tube with a $360 \mu \mathrm{m}$ inner diameter, using a microfluidic fitting and Upchurch connectors. The syringe was controlled with a Harvard Syringe pump to ensure reproducibility of the liquid meniscus position before turning on the laser. 


\section{APPENDIX C: CONICAL INDENTER DETAILS}

The indenter (solid needle in our case) is impressed into the surface of the agarose gel using a known applied force, as shown in Fig. 12(a).

During the early stages of indentation $(t \leq 1 \mathrm{~ms})$, the water in the agarose gel does not have sufficient time to flow away, and the gel behaves as an incompressible elastic solid. The instantaneous force $F_{0}$ correlates with the shear modulus $G$ of the gel $^{51}$ as

$$
F_{0}=4 G r \delta
$$

where $\delta$ corresponds to the indenter tip position with respect to the gel surface and $r$ is the radius of the immersed indenter volume, as shown in Fig. 12(a). After this time, the solvent can flow away and the agarose gel starts to relax, the force reaches a threshold value $F_{\infty}$, and the gel behaves as a compressible elastic solid. The qualitative force behavior is plotted in Fig. 12(b).

The maximum deformation $\delta$ caused by the needle tip displacement is plotted in Fig. 13(b), blue dots. The expected needle position without the agarose gel $y_{\text {needle }}$ is taken from the calibration process described in Sec. II A, green line. As we expected,
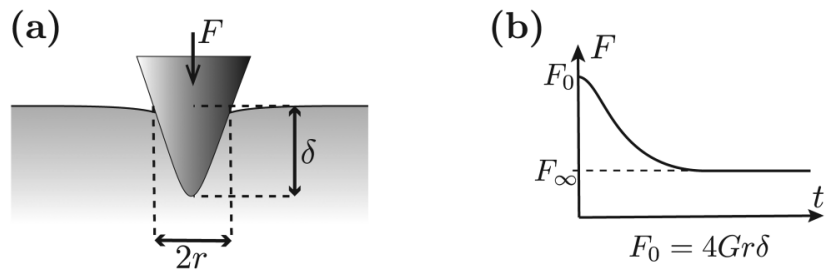

(c)
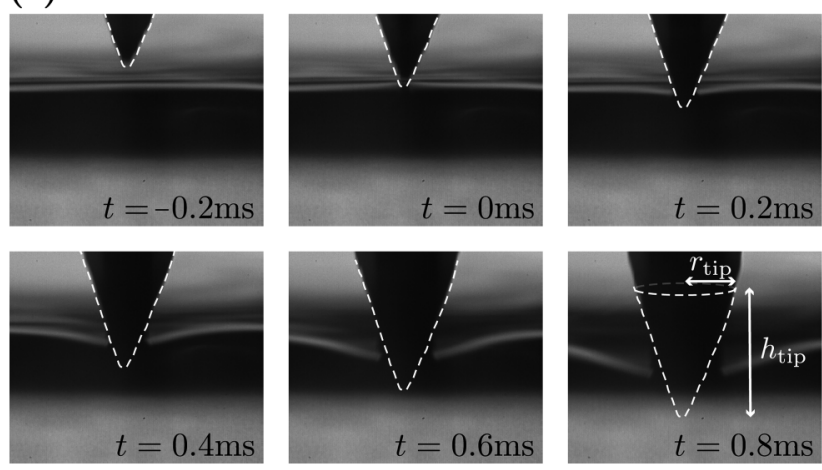

FIG. 12. (a) A schematic representation of the conical indentation test, where $\delta$ corresponds to the indenter tip position with respect to the gel surface and $r$ is the radius of the impressed cone volume. (b) Force relaxation in time during the indentation test. An initial instantaneous force $F_{0}$ is exerted by the gel opposing the indenting force, which directly relates to the shear modulus $G$ as $F_{0}=4 \mathrm{Gr} \delta$. (c) Image sequence of the needle solid injection process before the skin surrogate rupture, the first needle-agarose contact occurs at $t=0 \mathrm{~s}$. The needle tip has a right circular conical surface, represented by the whitedashed line, with a height and a base radius of $h_{\text {tip }}=0.174 \mathrm{~mm}$ and $r_{\text {tip }}=0.068 \mathrm{~mm}$, respectively.
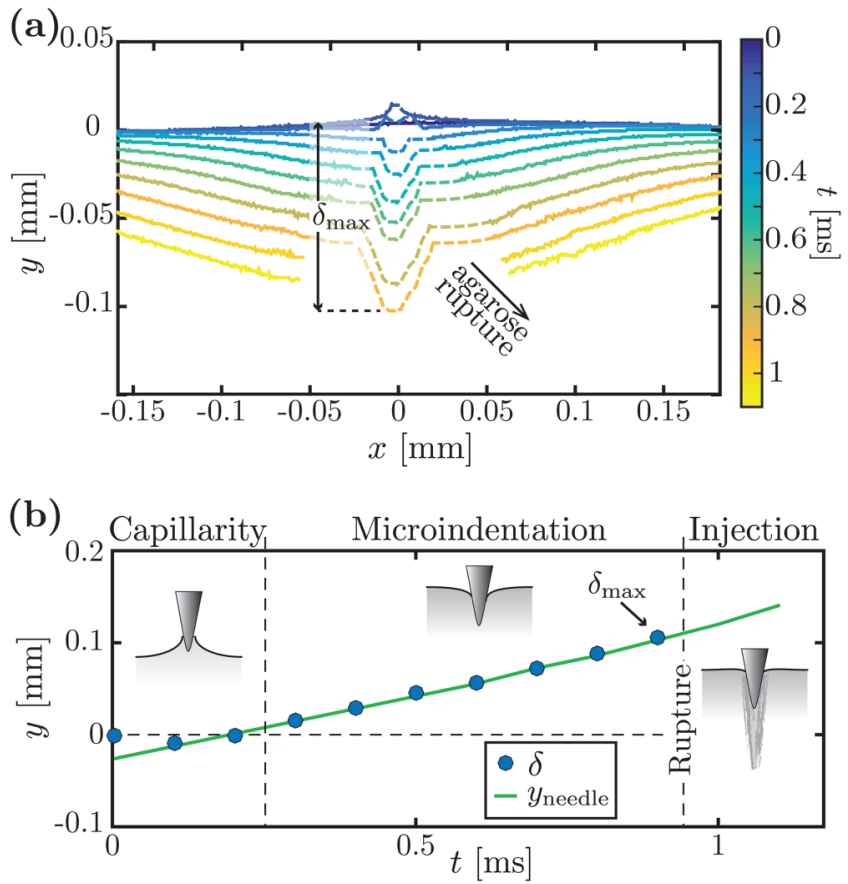

FIG. 13. (a) Skin surrogate surface deformation during solid needle microindentation. The maximum deformation just before rupture $\delta_{\max }$ is shown. (b) Maximum deformation $\delta$ and expected needle tip position $y_{\text {needle }}$ vs time. The three stages of the surface deformation (capillarity, microindentation, and rupture) are sketched and delimited.

$\delta$ and $y_{\text {needle }}$ are in perfect agreement, which means that the force exerted by the agarose gel is negligible compared to that exerted by the injector, meaning that the needle displacement is not affected by the gel.

\section{REFERENCES}

${ }^{1}$ K. Sperry, "Tattoos and tattooing. Part I: History and methodology," Am. J. Forensic Med. Pathol. 12(4), 313-319 (1991).

${ }^{\mathbf{2}}$ N. Kluger and V. Koljonen, "Tattoos, inks, and cancer," Lancet Oncol. 13(4), e161-e168 (2012).

${ }^{3}$ P. S. Islam, C. Chang, C. Selmi, E. Generali, A. Huntley, S. S. Teuber, and M. E. Gershwin, "Medical complications of tattoos: A comprehensive review," Clin. Rev. Allergy Immunol. 50(2), 273-286 (2016).

${ }^{4}$ R. Friedman, D. Antoine, S. Talamo, P. J. Reimer, J. H. Taylor, B. Wills, and M. A. Mannino, "Natural mummies from predynastic Egypt reveal the world's earliest figural tattoos," J. Archaeol. Sci. 92, 116-125 (2018).

${ }^{5}$ J. Serup, N. Kluger, and W. Bäumler, Tattooed Skin and Health, Current Problems in Dermatology (Karger Medical and Scientific Publishers, 2015).

${ }^{6}$ P. Piccinini, S. Pakalin, L. Contor, I. Bianchi, and C. Senaldi, "Safety of tattoos and permanent make-up: Final report," JRC Science for Policy Report EUR, Vol. 27947, 2016.

${ }^{7}$ H. Petersen and K. Roth, "To tattoo or not to tattoo," Chem. Unserer Zeit 50(1), 44-66 (2016) (English translation from www.chemistryviews.org).

${ }^{8}$ D. Jang, D. Kim, and J. Moon, "Influence of fluid physical properties on ink-jet printability," Langmuir 25(5), 2629-2635 (2009). 
${ }^{9}$ C. A. Grant, P. C. Twigg, R. Baker, and D. J. Tobin, "Tattoo ink nanoparticles in skin tissue and fibroblasts," Beilstein J. Nanotechnol. 6, 1183 (2015).

${ }^{10}$ Y.-C. Kim, Skin Vaccination Methods: Gene Gun, Jet Injector, Tattoo Vaccine, and Microneedle (Springer, Berlin, 2017), pp. 485-499.

${ }^{1}$ A. Taberner, N. C. Hogan, and I. W. Hunter, "Needle-free jet injection using real-time controlled linear Lorentz-force actuators," Med. Eng. Phys. 34(9), $1228-1235$ (2012)

${ }^{12} \mathrm{X}$. Li, B. Ruddy, and A. Taberner, "Characterization of needle-assisted jet injections," J. Control Release 243, 195-203 (2016).

${ }^{13}$ A. Arora, M. R. Prausnitz, and S. Mitragotri, "Micro-scale devices for transdermal drug delivery," Int. J. Pharm. 364(2), 227-236 (2008).

${ }^{14}$ R. Vecchione, S. Coppola, E. Esposito, C. Casale, V. Vespini, S. Grilli, P. Ferraro, and P. A. Netti, "Electro-drawn drug-loaded biodegradable polymer microneedles as a viable route to hypodermic injection," Adv. Funct. Mater. 24(23), 3515-3523 (2014)

${ }^{15} \mathrm{~S}$. Mitragotri, "Current status and future prospects of needle-free liquid jet injectors," Nat. Rev. Drug Discov. 5, 543-548 (2006).

${ }^{16}$ A. Arora, Liquid and Powder Jet Injectors in Drug Delivery: Mechanisms, Designs, and Applications (Springer, Berlin, 2017), pp. 221-230.

${ }^{17}$ S. Münch, J. Wohlrab, and R. Neubert, "Dermal and transdermal delivery of pharmaceutically relevant macromolecules," Eur. J. Pharm. Biopharm. 119, 235-242 (2017).

${ }^{18} \mathrm{~J}$. Baxter and S. Mitragotri, "Jet-induced skin puncture and its impact on needle-free jet injections: Experimental studies and a predictive model," J. Controlled Release 106(3), 361-373 (2005).

${ }^{19} \mathrm{~J}$. Schramm and S. Mitragotri, "Transdermal drug delivery by jet injectors: Energetics of jet formation and penetration," Pharm. Res. 19(11), 1673-1679 (2002).

${ }^{\mathbf{2 0}} \mathrm{G}$. Nelmes, "The peace gun and the eradication of smallpox," Mil. Med. 182(3-4), 1512-1513 (2017).

${ }^{21}$ N. C. Hogan, A. J. Taberner, L. A. Jones, and I. W. Hunter, "Needle-free delivery of macromolecules through the skin using controllable jet injectors," Expert Opin. Drug Deliv. 12(10), 1637-1648 (2015).

${ }^{22}$ P. Ferraro, S. Coppola, S. Grilli, M. Paturzo, and V. Vespini, "Dispensing nano-pico droplets and liquid patterning by pyroelectrodynamic shooting," Nat. Nanotechnol. 5(6), 429 (2010).

${ }^{23}$ Y. Tagawa, N. Oudalov, C. W. Visser, I. R. Peters, D. van der Meer, C. Sun, A. Prosperetti, and D. Lohse, "Highly focused supersonic microjets," Phys. Rev. X 2(3), 031002 (2012)

${ }^{24}$ Y. Tagawa, A. E. Oudalov, N. Ghalbzouri, C. Sun, and D. Lohse, "Needle-free injection into skin and soft matter with highly focused microjets," Lab Chip 13, 1357-1363 (2013).

${ }^{25}$ O. Gennari, L. Battista, B. Silva, S. Grilli, L. Miccio, V. Vespini, S. Coppola, P. Orlando, L. Aprin, P. Slangen et al., "Investigation on cone jetting regimes of liquid droplets subjected to pyroelectric fields induced by laser blasts," Appl. Phys. Lett. 106(5), 054103 (2015).

${ }^{26}$ S. F. Rastopov and A. T. Sukhodolsky, "Sound generation by thermocavitationinduced CW laser in solutions," in Optical Radiation Interaction with Matter (International Society for Optics and Photonics, 1991), Vol. 1440, pp. 127-135.

${ }^{27}$ J. P. Padilla-Martinez, C. Berrospe-Rodriguez, G. Aguilar, J. C. Ramirez-SanJuan, and R. Ramos-Garcia, "Optic cavitation with CW lasers: A review," Phys. Fluids 26, 122007 (2014)

${ }^{28}$ C. Berrospe-Rodriguez, C. W. Visser, S. Schlautmann, R. Ramos-Garcia, and D. Fernandez Rivas, "Continuous-wave laser generated jets for needle free applications," Biomicrofluidics 10(1), 014104 (2016).
${ }^{29}$ C. B. Rodríguez, C. W. Visser, S. Schlautmann, D. F. Rivas, and R. Ramos-Garcia, "Toward jet injection by continuous-wave laser cavitation," J. Biomed. Opt. 22(10), 105003 (2017).

${ }^{30}$ J. Serup, N. Kluger, and W. Bäumler, Tattooed Skin and Health, Tattoo Machines, Needles and Utilities (Karger Medical and Scientific Publishers, 2015).

${ }^{31} \mathrm{H}$. Wijshoff, "The dynamics of the piezo inkjet printhead operation," Phys. Rep. 491(4), 77-177 (2010).

${ }^{32}$ W. J. Grande, "Direct capillary printing in medical device manufacture," in Medical Coatings Deposition Technologies (John Wiley \& Sons, 2016), pp. 309-372.

${ }^{33} \mathrm{M}$. A. Kendall, "The delivery of particulate vaccines and drugs to human skin with a practical, hand-held shock tube-based system," Shock Waves 12(1), 23-30 (2002).

${ }^{34}$ Y. Deng, G. Winter, and J. Myschik, "Preparation and validation of a skin model for the evaluation of intradermal powder injection devices," Eur. I. Pharm. Biopharm. 81(2), 360-368 (2012).

${ }^{35}$ J. Schramm-Baxter, J. Katrencik, and S. Mitragotri, "Jet injection into polyacrylamide gels: Investigation of jet injection mechanics," J. Biomech. 37(8), 1181-1188 (2004).

${ }^{36} \mathrm{R}$. Williams, "Jet injection of viscous fluids," Ph.D. thesis (ResearchSpace at University of Auckland, 2016).

${ }^{37} \mathrm{M}$. Ahearne, Y. Yang, A. J. El Haj, K. Y. Then, and K.-K. Liu, "Characterizing the viscoelastic properties of thin hydrogel-based constructs for tissue engineering applications," J. R. Soc. Interface 2(5), 455-463 (2005).

${ }^{38} \mathrm{Y}$. Hu, X. Zhao, J. J. Vlassak, and Z. Suo, "Using indentation to characterize the poroelasticity of gels," Appl. Phys. Lett. 96(12), 121904 (2010).

${ }^{39}$ D. M. Ebenstein and L. A. Pruitt, "Nanoindentation of soft hydrated materials for application to vascular tissues," J. Biomed. Mater. Res. Part A 69A(2), 222-232 (2004).

${ }^{40} \mathrm{G}$. Constantinides, Z. I. Kalcioglu, M. McFarland, J. F. Smith, and K. J. V. Vliet, "Probing mechanical properties of fully hydrated gels and biological tissues," J. Biomech. 41(15), 3285-3289 (2008).

${ }^{41}$ C.-Y. Hui, Y. Y. Lin, F.-C. Chuang, K. R. Shull, and W.-C. Lin, "A contact mechanics method for characterizing the elastic properties and permeability of gels," J. Polym. Sci. Part B: Polym. Phys. 44(2), 359-370 (2006).

${ }^{42}$ M. Galli, K. S. Comley, T. A. Shean, and M. L. Oyen, "Viscoelastic and poroelastic mechanical characterization of hydrated gels," J. Mater. Res. 24(3), 973 (2009).

${ }^{43}$ S. Chen and A. Soh, "The capillary force in micro- and nano-indentation with different indenter shapes," Int. J. Solids Struct. 45(10), 3122-3137 (2008).

${ }^{44} \mathrm{Y}$. Men, X. Zhang, and W. Wang, "Capillary liquid bridges in atomic force microscopy: Formation, rupture, and hysteresis," J. Chem. Phys. 131(18), 184702 (2009).

${ }^{45} \mathrm{~V}$. Normand, D. L. Lootens, E. Amici, K. P. Plucknett, and P. Aymard, "New insight into agarose gel mechanical properties," Biomacromolecules 1(4), 730-738 (2000).

${ }^{46} \mathrm{~V}$. Nayar, J. Weiland, C. Nelson, and A. Hodge, "Elastic and viscoelastic characterization of agar,” J. Mech. Behav. Biomed. Mater. 7, 60-68 (2012).

${ }^{47} \mathrm{~L}$. Landau and B. Levich, "Dragging of a liquid by a moving plate," in Dynamics of Curved Fronts (Academic Press, San Diego, CA, 1988), pp. 141-153.

${ }^{48}$ E. Rio and F. Boulogne, "Withdrawing a solid from a bath: How much liquid is coated?," Adv. Colloid Interface Sci. 247, 100-114 (2017).

${ }^{49}$ Several tattooing and PMU companies, personal communication (2015-2018).

${ }^{50}$ I. Weijers, Design for a Needle Free Tattoo Machine for Permanent Make Up (University of Twente, 2017).

${ }^{51}$ K. L. Johnson, Contact Mechanics (Cambridge University Press, 1985). 